\title{
Determination of the prevalence of lymphatic filariasis among migrant workers in Kuwait by detecting circulating filarial antigen
}

\begin{abstract}
Correspondence
Jamshaid lqbal

iqbal@hsc.edu.kw
\end{abstract}

Received 16 October 2005

Accepted 7 December 2005

\author{
Jamshaid Iqbal ${ }^{1}$ and Ali Sher ${ }^{2}$ \\ ${ }^{1}$ Department of Microbiology, Faculty of Medicine, Kuwait University, PO Box 24923, \\ Safat 13110, Kuwait \\ ${ }^{2}$ Malaria Laboratory, Ports and Borders Center, Ministry of Health, Kuwait
}

\section{INTRODUCTION}

Lymphatic filariasis continues to be a major source of permanent disability and an impediment to socio-economic development in 73 countries where more than 1 billion people are at risk and over 120 million are infected. Wuchereria bancrofti is responsible for approximately $90 \%$ of infections (World Health Organization, 1999).

Early and efficient diagnosis of $W$. bancrofti infection is a key step in monitoring, treatment and subsequent eradication of lymphatic filariasis. Microscopic examination of a thick blood film or nucleopore-filtered blood (NFB) to detect microfilariae ( $\mathrm{mf}$ ) lacks sensitivity and provides only an indirect measure of the adult worm load. Moreover, the current filarial antibody detection tests remain unable to distinguish between active and past infections (Bruschi \& Castagna, 2004).

Abbreviations: $\mathrm{AU}$, antigen units; $\mathrm{CFA}$, circulating filarial antigen; ICT, immunochromatographic test; NFB, nucleopore-filtered blood; mf, microfilariae; ML, maximum likelihood.
Recently, an immunochromatographic test (ICT) using whole-blood samples was introduced. The test detects soluble circulating filarial antigen (CFA) in blood (McCarthy, 2000; Weerasooriya et al., 2003). The ICT has been evaluated for its sensitivity and specificity in various epidemiological settings and the reported sensitivity ranges from 73 to $100 \%$ (Braga et al., 2003; Chandrasena et al., 2002; Omar et al., 2000; Reddy et al., 2004).

No indigenous case of filarial infection in Kuwait has been reported as yet; however, the presence of Culex quinquefasciatus, the vector of bancroftian filariasis, and a large number of potentially infected individuals in Kuwait may initiate local transmission. Every year, more than 45000 migrant workers arrive in Kuwait from areas where filarial infection is endemic. On arrival in Kuwait, these workers are screened for filarial infection, malaria, hepatitis, human immunodeficiency virus and tuberculosis infection before they are issued a resident permit. Screening for filarial infection is done by microscopy of a thick blood film. Due to technical, logistical and administrative constraints, these 
workers can only be screened during the daytime, although $\mathrm{mf}$ are present mostly in the blood circulation at night. This study was carried out to determine the level of filarial infection among migrant workers in Kuwait by detecting filarial antigen using the ICT and the TropBio assay.

\section{METHODS}

Study population. The study was performed at the Ports and Borders Center, Ministry of Health, Kuwait, and the Department of Microbiology, Faculty of Medicine, Kuwait University, during the period April 2000 to November 2003. A total of 1050 immigrant workers from countries where filarial infection is endemic were selected randomly and enrolled in the study. The majority (>95\%) of these individuals were from India (Andhra Pradesh, Bihar, Tamil Nadu and Uttar Pradesh - the filaria belt), the Philippines and Sri Lanka. The others were from Tanzania, Mali, Gambia and Sudan. For comparison, control blood specimens were also collected from 260 individuals residing in Kuwait comprising 50 healthy Kuwaiti blood donors, 50 individuals originally from endemic areas who had been living in Kuwait for more than 10 years and had tested negative repeatedly for $\mathrm{mf}$, and 160 patients with other parasitic infections (hydatid disease, amoebiasis, malaria, schistosomiasis) from areas non-endemic for filaria. Socio-demographic information and their history of travel were collected from all individuals enrolled in the study.

All specimens were tested blindly at the Ports and Borders Center, Kuwait, and the Department of Microbiology, Faculty of Medicine, Kuwait University. All participants were informed about the objectives of the study and their consent was obtained. The Ethical Committee of the Faculty of Medicine approved the study.

Specimen collection. Due to administrative and logistical reasons, all blood specimens were collected between 8 a.m. and 10 a.m. Blood $(2 \mathrm{ml})$ was collected into an EDTA-coated tube and tests were performed on the same day. Blood specimens were also collected at midnight from 25 microscopy-negative but ICT-positive individuals for retesting of filtered blood by microscopy.

NFB microscopy. The blood was diluted with $9 \mathrm{ml} 2 \%$ formalin in normal saline and filtered using a nucleopore polycarbonate membrane (pore size $5 \mu \mathrm{m}$, diameter $25 \mathrm{~mm}$ ). The filter membranes were stained with Giemsa stain and examined by two experienced microscopists under low-power magnification for the presence of $\mathrm{mf}$.

ICT. The ICT uses paired antibodies (polyclonal and monoclonal antibodies) specific for $W$. bancrofti antigen (AD12.1) conjugated to visible colloidal gold particles and immobilized on the test strip. The test was performed as described by the manufacturers (NOW ICT Filariasis Test; Binax). Briefly, $50 \mu$ whole blood was added to the sample pad, allowing any filarial antigen present in the plasma to bind to the colloidal gold-labelled antibody on the strip. Cards exhibiting the control line were considered valid. Reading and interpretation of the results followed the manufacturer's instructions.

TropBio ELISA assay. The TropBio ELISA assay (JCU Tropical Biotechnology) detects and quantifies $W$. bancrofti antigen in blood specimens. The assay was performed as described previously (Weerasooriya et al., 2003). Briefly, each specimen was tested in duplicate. The mean of the results was classified against seven known standard concentrations of Onchocerca gibsoni antigen and a standard curve was produced relating absorbance and antigen content. The antigen concentration and the antigen titre group for each specimen were determined from the standard curve. A cut-off value of 32 antigen units (AU) (titre group 4) was considered positive for CFA and a value of $>8000$ AU was assigned to titre group 7 .
Data analysis. The sensitivity of NFB microscopy and the ICT is unknown, as many individuals may harbour adult worms without having $\mathrm{mf}$ in their peripheral blood. Therefore, we assumed that both NFB microscopy and the ICT might be $100 \%$ specific, but not $100 \%$ sensitive. Making this assumption, the sensitivity and specificity of the NFB microscopy and the ICT and the prevalence of filarial infection among the immigrant workers were calculated using statistical maximum-likelihood (ML) methods (Goldberg \& Wittes, 1978) and the specificity by using the equation derived by Staquet et al. (1981).

\section{RESULTS}

The presence of filarial infection was examined in 1050 migrant workers $[603(57 \cdot 4 \%)$ males and $447(42 \cdot 6 \%)$ females aged between 14 and 61 years (mean \pm SD 29.2 \pm $3 \cdot 2$ )] by NFB microscopy and for the presence of CFA using the ICT and the TropBio assay. The overall prevalence of filarial antigenaemia in this group was $18.3 \%$ (192 individuals) using the ICT and $20 \cdot 3 \%$ (213 individuals) using the TropBio assay (Table 1). However, microfilaraemia was present only in 34 cases (3\%): 19 cases had $<100 \mathrm{mf} \mathrm{ml}^{-1}$, 10 cases had $100-750 \mathrm{mf} \mathrm{ml}^{-1}$ and five cases had $>750 \mathrm{mf}$ $\mathrm{ml}^{-1}$. The mean $\mathrm{mf}$ count in these cases was $816 \mathrm{mf} \mathrm{ml}^{-1}$ (range: 17-1283 $\mathrm{mf} \mathrm{m}^{-1}$ ). Only two cases of Brugia malayi were observed; both of these cases were from the Philippines. These two cases were excluded from further analysis by the ICT and the TropBio assay. All other cases had W. bancrofti infections and were also positive for CFA using the ICT and the TropBio assay. Given the logistical constraints of collecting midnight blood specimens from all subjects, a small group of 25 NFB-negative, ICT-positive individuals was retested by taking their blood specimens at night. Only two of these 25 individuals showed $\mathrm{mf}$ in NFB in their midnight specimens; the density of $\mathrm{mf}$ in these two cases was $<50 \mathrm{mf} \mathrm{ml}^{-1}$ (data not shown). Only two of the 260 control specimens were positive for CFA using the ICT and the TropBio assay (Table 1). Both of these cases were males from Andhra Pradesh, India, working in Kuwait and had not travelled to India in the last 5 years. These cases were excluded in the determination of test specificity. The majority of cases $(>90 \%)$ with antigenaemia and microfilaraemia were males in the $24-41$ year age group.

The performance of the ICT in detecting antigenaemia was compared with NFB microscopy (Table 2). Among 192 cases with CFA, 30 cases $(15 \cdot 6 \%)$ had microfilaraemia. However, two mf-positive cases with a density of $<20 \mathrm{mf} \mathrm{ml}^{-1}$ did not show any detectable CFA (Table 2). The sensitivities of NFB microscopy and the ICT and the prevalence of infection among the immigrant workers were calculated using the ML statistical method. The prevalence of filarial infection in the migrant population was estimated to be $19.5 \%$; the sensitivity of NFB microscopy was only $15 \cdot 9 \%$, whilst the sensitivity of the ICT was $93.8 \%$. The specificity of the ICT ranged from $84 \cdot 1$ to $100 \%$, based on the prevalence of infection using NFB microscopy and the ICT, calculated as described in Methods.

The TropBio assay gives a quantitative response expressed in AU or titre groups. Of 213 cases showing various levels of 
Table 1. Characteristics of study individuals and the number of cases positive for filarial infection by NFB microscopy, the TropBio assay and the ICT

\begin{tabular}{|lccccc|}
\hline $\begin{array}{l}\text { Categories of } \\
\text { study cases }\end{array}$ & $\begin{array}{c}\text { No. tested } \\
\text { (males/females) }\end{array}$ & $\begin{array}{c}\text { Mean age in } \\
\text { years (range) }\end{array}$ & \multicolumn{2}{c|}{ No. of positive cases } \\
\cline { 4 - 6 } & & & $\begin{array}{c}\text { NFB } \\
\text { microscopy }\end{array}$ & ICT & TropBio \\
\hline Immigrants & $1050(603 / 447)$ & $29 \cdot 2(14-61)$ & $34^{*}$ & 192 & 213 \\
Endemic mf-negative $\dagger$ & $50(30 / 20)$ & $31 \cdot 5(5-59)$ & 0 & 2 & 2 \\
Non-endemic mf-negative & $50(25 / 25)$ & $25 \cdot 1(21-49)$ & 0 & 0 & 0 \\
Other parasitic infections $\ddagger$ & & & & & \\
$\quad$ Malaria & $50(23 / 27)$ & $32 \cdot 1(14-54)$ & 0 & 0 & 0 \\
Hydatid disease & $30(21 / 9)$ & $33 \cdot 4(23-58)$ & 0 & 0 & 0 \\
Amoebic abscess & $20(14 / 6)$ & $29 \cdot 2(21-54)$ & 0 & 0 & 0 \\
Schistosomiasis & $60(35 / 25)$ & $28 \cdot 6(26-67)$ & 0 & 0 & 0 \\
\hline
\end{tabular}

${ }^{\star}$ Two cases had B. malayi $\mathrm{mf}$ and thus were excluded from further analysis.

$\dagger$ Individuals were from filaria-endemic areas and were negative for mf by NFB microscopy.

‡Groups of migrants in Kuwait who had never been exposed to filarial infection and were negative for mf by microscopy but positive for other parasitic infections.

CFA, 75 had CFA levels of 32-128 AU (group 4), 48 had CFA up to 512 AU (group 5), 71 had CFA up to 2048 AU (group 6) and 19 had CFA > 8000 AU (group 7). Analysis of the relationship between CFA levels and $\mathrm{mf}$ density showed a positive association, but was not statistically significant $(P>0 \cdot 05)$.

The performance of the TropBio assay for detection of CFA showed a similar pattern to that of the ICT (Table 2). The ICT detected CFA in $192(90 \cdot 1 \%)$ of the 213 cases that were positive with the TropBio assay. Comparing these two tests, the sensitivity of the ICT was $92 \cdot 1 \%$ (Table 2 ). Furthermore, the overall sensitivity of the ICT and the TropBio assay for detection of CFA in cases with different $\mathrm{mf}$ densities was comparable.

\section{DISCUSSION}

The recent development of reliable tests for detection of CFA in W. bancrofti-infected individuals has been highly successful. Several studies have reported the sensitivity, specificity and predictive values of the ICT in different epidemiological settings (Braga et al., 2003; Chandrasena et al., 2002; Omar et al., 2000). In this study, the sensitivity of the ICT was $93 \cdot 8 \%$. Contrary to most previous studies, the performance of the ICT, the TropBio assay and NFB microscopy was determined in all study subjects in this study. The imperfect 'gold standards' like NFB microscopy generally introduce bias, such as an underestimation of the prevalence of filarial infection, specificity and the positive predictive value of the new test. Therefore, in this study, all statistical analysis was done using ML methods and Staquet's equation (Goldberg \& Wittes, 1978; Staquet et al., 1981); these are considered to be the ideal methods for calculation of sensitivity and specificity of new screening assays.

The overall prevalence of filarial antigenaemia among migrant workers was $18 \cdot 3 \%$ and microfilaraemia was present only in $3 \%$ of cases. Since mf are present mostly in the blood circulation at night, the number of microfilaraemic individuals detected using daytime blood samples was expected to be significantly lower than that obtained with night-time blood samples. However, as mentioned above, night-time blood samples were not available due to unavoidable administrative and technical reasons. Given the logistical

Table 2. Performance of NFB microscopy, the ICT and the TropBio assay in detecting filarial infection in the migrant population in Kuwait

Sensitivity and specificity were calculated using ML methods and Staquet's equation as described in Methods.

\begin{tabular}{|lcrrrr|}
\hline \multirow{2}{*}{$\begin{array}{l}\text { ICT results } \\
\text { (no. of cases) }\end{array}$} & \multicolumn{2}{c}{ NFB microscopy } & & \multicolumn{2}{c|}{ TropBio assay } \\
\cline { 2 - 3 } & Pos. (\%) & Neg. (\%) & & Pos. (\%) & Neg. (\%) \\
\hline Positive (192) & $30(93 \cdot 8)$ & $162(15 \cdot 9)$ & & $192(90 \cdot 1)$ & 0 \\
Negative (858) & $2(6 \cdot 2)$ & $856(84 \cdot 1)$ & & $21(9 \cdot 9)$ & $837(100)$ \\
Total $(1050)$ & $32(100)$ & $1018(100)$ & & $213(100)$ & $837(100)$ \\
\hline
\end{tabular}


and technical constraints, night-time blood specimens were only collected from 25 NFB-negative subjects with CFA and retested for $\mathrm{mf}$ by microscopy. Only two of these 25 subjects showed $\mathrm{mf}$ with a density of $<50 \mathrm{mf} \mathrm{ml}^{-1}$. More than $90 \%$ of our study subjects were from filariaendemic areas (Andhra Pradesh, Bihar, Tamil Nadu and Uttar Pradesh - the filaria belt) in India. The microfilaria infection rate in our study correlated well with that reported from these areas (World Health Organization, 1999); however, the prevalence of CFA in our study group was much higher than that of $10.6 \%$ observed in the Indian expatriate population in Saudi Arabia (Omar et al., 2000). This is probably due to inclusion of groups of individuals from Kerala in the Saudi study, where filarial infection is very low. In this study, $162(15.9 \%) \mathrm{mf}$-negative individuals showed the presence of CFA using the ICT. However, the ICT failed to detect two of the $32 \mathrm{mf}$-positive cases. The microfilaria load in both of these cases was $<20 \mathrm{mf} \mathrm{ml}^{-1}$, suggesting a decreased sensitivity of the ICT in cases with a low $\mathrm{mf}$ density. The low sensitivity of the ICT was reported previously from India in cases with low $\mathrm{mf}$ density (Sunish et al., 2001). We observed an overall sensitivity of $93 \cdot 8 \%$ for the ICT.

The estimated value for specificity of the ICT ranged from 84 to $100 \%$ based on the prevalence estimated by NFB microscopy and the ICT. Microscopy detects only infections with patent $\mathrm{mf}$ circulating during the night. However, asymptomatic amicrofilaraemic infections have also been well documented (Eigege et al., 2003). Thus, the 'false positives' obtained by the ICT could well be true positives due to absence of $\mathrm{mf}$ in the daytime blood samples or the presence of adult worms in the deep tissues. Similarly, the TropBio assay, which detects a different CFA, also detected a much higher prevalence of CFA than $\mathrm{mf}(20.3 \mathrm{vs} 3 \%$, respectively). Earlier studies have also reported similar results (Weerasooriya et al., 2003). There are several possible explanations for the mf-negative status of these infections. Some infections may be at the pre-patent stage and may be a single-sex infection. Another possibility is that a low level of adult worm infection produces antigenaemia but no detectable microfilaraemia (Eigege et al., 2003; Molyneux \& Taylor, 2001). Furthermore, several studies have reported that a considerable number of individuals remain antigenpositive for up to 2 years after diethylcarbamazine treatment (Eigege et al., 2003; Hoerauf, 2003; Meyrowitsch et al., 2004). However, recently one study reported a significant decrease in CFA levels at 18 months after diethylcarbamazine treatment among Myanmar migrants in Thailand (Koyadun et al., 2003).

The performance of the ICT and the TropBio assay to detect CFA showed a statistically significant correlation (192 cases vs 213 cases, respectively); a similar observation has been reported previously (Molyneux \& Taylor, 2001).

Although the TropBio assay provides a semi-quantitative response, probably reflecting the adult worm load, it requires more elaborate laboratory facilities and a well-equipped technical staff. The ICT requires minimal laboratory and technical support and thus is more suitable for diagnosis of filarial infection in clinical laboratories and for surveillance studies. In conclusion, this study demonstrated the high specificity and sensitivity of the ICT for detection of filarial infection. The ICT should be used to screen migrant workers in Kuwait for filarial infection using daytime blood specimens.

\section{ACKNOWLEDGEMENTS}

The authors thank the patients who participated in the study. The technical and logistical support provided by the Ports and Borders Center is appreciated. We thank Dr Saeed, Department of Community Medicine, Kuwait University, Kuwait, for assistance in statistical analysis of the data. The study was supported by a financial grant from the Research Administration, Kuwait University (MI 06/01).

\section{REFERENCES}

Braga, C., Dourado, M. I., Ximenes, R. A., Alves, L., Brayner, F., Rocha, A. \& Alexander, N. (2003). Field evaluation of the whole blood immunochromatographic test for rapid bancroftian filariasis diagnosis in the northeast of Brazil. Rev Inst Med Trop Sao Paulo 45, 125-129.

Bruschi, F. \& Castagna, B. (2004). The serodiagnosis of parasitic infections. Parassitologia 46, 141-144.

Chandrasena, T. G., Premaratna, R., Abeyewickrema, W. \& de Silva, N. R. (2002). Evaluation of the ICT whole-blood antigen card test to detect infection due to Wuchereria bancrofti in Sri Lanka. Trans $R$ Soc Trop Med Hyg 96, 60-63.

Eigege, A., Richards, F. O., Blaney, D. D. \& 8 other authors (2003). Rapid assessment for lymphatic filariasis in Central Nigeria: a comparison of the immunochromatographic card test and hydrocele rates in an area of high endemicity. Am J Trop Med Hyg 68, 643-646.

Goldberg, J. D. \& Wittes, J. T. (1978). The estimation of false negatives in medical screening. Biometrics 34, 77-86.

Hoerauf, A. (2003). Control of filarial infections: not the beginning of the end, but more research is needed. Curr Opin Infect Dis 16, 403-410.

Koyadun, S., Bhumiratana, A. \& Prikchu, P. (2003). Wuchereria bancrofti antigenemia clearance among Myanmar migrants after biannual mass treatments with diethylcarbamazine, $300 \mathrm{mg}$ oral-dose FILADEC tablet, in Southern Thailand. Southeast Asian J Trop Med Public Health 34, 758-767.

McCarthy, J. (2000). Diagnosis of lymphatic filariasis infection. In Lymphatic Filariasis, pp. 127-150. Edited by T. B. Nutman. London: Imperial College Press.

Meyrowitsch, D. W., Simonsen, P. E. \& Magesa, S. M. (2004). Longterm effect of three different strategies for mass diethylcarbamazine administration in bancroftian filariasis: follow-up at 10 years after treatment. Trans R Soc Trop Med Hyg 98, 627-634.

Molyneux, D. H. \& Taylor, M. J. (2001). Current status and future prospects of the Global Lymphatic Filariasis Programme. Curr Opin Infect Dis 14, 155-159.

Omar, M. S., Sheikha, A. K., Al-Amari, O. M., Abdalla, S. E. \& Musa, R. A. (2000). Field evaluation of two diagnostic antigen tests for Wuchereria bancrofti infection among Indian expatriates in Saudi Arabia. Southeast Asian J Trop Med Public Health 31, 415-418.

Reddy, G. S., Das, L. K. \& Pani, S. P. (2004). The preferential site of adult Wuchereria bancrofti: an ultrasound study of male asymptomatic 
microfilaria carriers in Pondicherry, India. Natl Med J India 17, 195-196.

Staquet, M., Rozencweig, M., Lee, Y. J. \& Muggia, F. M. (1981). Methodology for the assessment of new dichotomous diagnostic tests. J Chronic Dis 34, 599-610.

Sunish, I. P., Rajendran, R., Satyanarayana, K., Munirathinam, A. \& Gajanana, A. (2001). Immunochromatographic test (ICT) for estimation of true prevalence of bancroftian filariasis in an endemic area in South India. Trans R Soc Trop Med Hyg 95, 607-609.
Weerasooriya, M. V., Itoh, M., Mudalige, M. P., Qiu, X. G., Kimura, E., Gunawardena, N. K. \& Fujimaki, Y. (2003). Human infection with Wuchereria bancrofti in Matara, Sri Lanka: the use in parallel, of an ELISA to detect filarial-specific IgG4 in urine and of ICT card tests to detect filarial antigen in whole blood. Ann Trop Med Parasitol 97, 179-185.

World Health Organization (1999). Can lymphatic filariasis and onchocerciasis be eliminated together? TDR News No. 60. Filarial Update. 1 (2). 